\title{
Coefficient-explicit Condition Number Bounds for Overlapping Additive Schwarz
}

\author{
I.G. Graham and R. Scheichl \\ Department of Mathematical Sciences, University of Bath, Bath BA2 7AY, United \\ Kingdom. Email: I.G.Graham@bath.ac.uk, R.Scheichl@bath.ac.uk
}

\section{Introduction}

In this paper we discuss new domain decomposition preconditioners for piecewise linear finite element discretisations of boundary-value problems for the model elliptic problem

$$
-\nabla \cdot(\mathcal{A} \nabla u)=f,
$$

in a bounded polygonal or polyhedral domain $\Omega \subset \mathbb{R}^{d}, d=2$ or 3 with suitable boundary data on the boundary $\partial \Omega$. The tensor $\mathcal{A}(x)$ is assumed isotropic and symmetric positive definite, but may vary with many orders of magnitude in an unstructured way on $\Omega$. Many examples arise in groundwater flow and oil reservoir modelling.

Let $\mathcal{T}^{h}$ be a conforming shape-regular simplicial mesh on $\Omega$ and let $\mathcal{S}^{h}(\Omega)$ denote the space of continuous piecewise linear finite elements on $\mathcal{T}^{h}$. The finite element discretisation of (1) in $\mathcal{V}^{h}$ (the $n$-dimensional subspace of functions in $\mathcal{S}^{h}(\Omega)$ which vanish on essential boundaries), yields the linear system:

$$
A \mathbf{u}=\mathbf{f},
$$

and it is well-known that the conditioning of $A$ worsens when $\mathcal{T}^{h}$ is refined or when the heterogeneity (characterised by the range of $\mathcal{A}$ ) becomes large. It is of interest to find solvers for (2) which are robust to changes in the mesh width $h$ as well as to the heterogeneity.

While there are many papers which solve (2) for "layered media" in which discontinuities in $\mathcal{A}$ are simple interfaces that can be resolved by a coarse mesh (see e.g. $[4,12]$ and the references therein), until recently there was no rigorously justified method for general heterogeneous media. We present here a summary of some recent papers $[6,7,10,11]$ where a new analysis of domain decomposition methods for (2) (which have inherent robustness with respect to $h$ ) was presented. This analysis indicates explicitly how subdomains and coarse spaces should be designed in order to achieve robustness also with respect to heterogeneities. More precisely this analysis introduces new "robustness indicators" (which depend on the choice of subdomains and coarse space 
and in particular depend on the energy of the coarse space basis functions) and proves that, if these indicators are controlled, then the preconditioner will be robust. Papers $[6,7]$ then go on to consider the use of multiscale finite elements to build coarse spaces for domain decomposition and prove a number of results which indicate their robustness in cases where standard coarsening methods fail to be robust. Papers $[10,11]$ consider aggregation-based coarsening (as introduced e.g. in [13,2]) and prove similar results as in the multiscale finite element case.

The coarse spaces proposed in [6] yield coefficient-dependent prolongation operators, similar to those which have been tested empirically in the context of (Schur complement based) domain decomposition methods in $[3,5]$. The concept of energy-minimising coarse spaces also appears in several papers on the construction of algebraic multigrid methods $[14,9,15]$, but their behaviour in the presence of heterogeneity is not analysed. The use of multiscale finite elements as coarseners was also proposed in [1], but again this was in the Schur-complement context and the analysis depended on classical periodic homogenisation theory. The analysis in [6] does not require periodicity and does not appeal to homogenisation theory. We are also not aware of any theoretical results which make explicit the dependency of the condition number on heterogeneities in $\mathcal{A}$ in the case of the aggregation-based coarse spaces proposed in [10].

Given a finite overlapping open covering of subdomains $\left\{\Omega_{i}: i=1, \ldots, s\right\}$ of $\Omega$, with each $\bar{\Omega}_{i}$ assumed to consist of a union of elements from $\mathcal{T}^{h}$, and a coarse basis $\left\{\Phi_{j}: j=1, \ldots, N\right\} \subset \mathcal{V}^{h}$, we study two-level additive Schwarz preconditioners

$$
M_{A S}^{-1}=\sum_{i=0}^{s} R_{i} A_{i}^{-1} R_{i}^{T} .
$$

Here, for $i=1, \ldots, s, R_{i}$ denotes the restriction matrix from freedoms in $\Omega$ to freedoms in $\Omega_{i}$ and $\left(R_{0}\right)_{j, p}=\Phi_{j}\left(x_{p}\right)$, where $x_{p}, p=1, \ldots, n$, are the interior nodes of the fine mesh $\mathcal{T}^{h}$. The matrices $A_{i}$ are then defined via the Galerkin product $A_{i}:=R_{i} A R_{i}^{T}$.

For the purposes of exposition we will only describe the theory for scalar $\mathcal{A}$ in (1), i.e. $\mathcal{A}=\alpha I$, and restrict to the case of homogeneous Dirichlet boundary conditions. For theoretical purposes, we shall also assume that $\alpha \geq 1$. This is no loss of generality, since problem (2) can be scaled by $\left(\min _{x} \alpha(x)\right)^{-1}$ without changing its conditioning. Throughout the paper, the notation $C \lesssim D$ (for two quantities $C, D$ ) means that $C / D$ is bounded above independently of the mesh parameters and of the coefficient function $\alpha$.

\section{Coefficient-explicit Schwarz Theory I}

The assumptions on the coarse space and on the overlapping subdomains made in the papers $[6,7]$ and $[10,11]$ are different. We start in this section with 
the theory presented in $[10,11]$. Although there we only applied the theory to aggregation-based coarsening, we will show here that it can also be applied in the case of the multiscale coarsening introduced in $[6,7]$, leading to a slightly different condition number bound than the one in $[6,7]$. The key assumption in this section is that the support of each coarse space basis function is fully contained in at least one subdomain. For details and proofs see [10].

We start with a linearly independent set $\left\{\Phi_{j}: j=1, \ldots, N_{H}\right\} \subset \mathcal{S}^{h}(\Omega)$. This set contains some functions which do note vanish on the boundary so $N_{H}>N$. We set $\omega_{j}=$ interior $\left(\operatorname{supp}\left\{\Phi_{j}\right\}\right)$ with diameter $H_{j}$. For theoretical purposes we assume that the $\left\{\omega_{j}\right\}$ form a shape-regular overlapping cover of $\Omega$ and that the overlap between any support $\omega_{j}$ and its neighbours is uniformly of size $\delta_{j}$. In addition we make the following assumptions:

(C1) For all $j=1, \ldots, N_{H}$ there is an $i_{j} \in\{1, \ldots, s\}$ such that $\omega_{j} \subset \Omega_{i_{j}}$.

(C2) $\sum_{j=1}^{N_{H}} \Phi_{j}(x)=1$, for all $x \in \bar{\Omega}$.

(C3) $\left\|\Phi_{j}\right\|_{L_{\infty}(\Omega)} \lesssim 1$.

We assume that the functions $\Phi_{j}$ are numbered in such a way that $\Phi_{j} \in \mathcal{V}^{h}$ for all $j \leq N$ and $\Phi_{j} \notin \mathcal{V}^{h}$ for all $j>N$. Thus we can denote the coarse space by $\mathcal{V}_{0}=\operatorname{span}\left\{\Phi_{j}: j=1, \ldots, N\right\}$ and we have $\mathcal{V}_{0} \subset \mathcal{V}^{h}$.

Note that although we have not directly made any assumptions on the overlap of the subdomains, (C1) implies that the overlap of a subdomain $\Omega_{i}$ and its neighbours is always bounded from below by $\min _{\left\{j: \omega_{j} \subset \Omega_{i}\right\}} \delta_{j}$.

It is known (see e.g. [12]) that in order to bound $\kappa\left(M_{A S}^{-1} A\right.$ ), we need to assume some upper bounds on $\left|\Phi_{j}\right|_{H^{1}(\Omega)}^{2}$. We take a novel approach here and introduce a quantity which also reflects how the coarse space handles the coefficient heterogeneity:

\section{Definition 1. (Coarse space robustness indicator I).}

$$
\gamma_{\infty}(\alpha)=\max _{j=1}^{N_{H}}\left\{\delta_{j}^{2}\left\|\alpha\left|\nabla \Phi_{j}\right|^{2}\right\|_{L_{\infty}(\Omega)}\right\} .
$$

The quantity $\gamma_{\infty}(\alpha)$ appears in our estimates for the two-level preconditioner below. Note that, roughly speaking, this robustness indicator is well-behaved if the $\Phi_{j}$ have small gradient wherever $\alpha$ is large. The weight $\delta_{i}^{2}$ is chosen to make $\gamma_{\infty}(\alpha) \lesssim 1$ when $\alpha=1$.

We can now state one of the main results from [10] (Theorem 3.8):

Theorem 1. Assume that (C1)-(C3) hold true. Then

$$
\kappa\left(M_{A S}^{-1} A\right) \lesssim \gamma_{\infty}(\alpha)\left(1+\max _{j=1}^{N_{H}} \frac{H_{j}}{\delta_{j}}\right)
$$

Example 1 (Linear Finite Element Coarsening). In the classical case, i.e. when $\left\{\Phi_{j}\right\}$ is the standard nodal basis for the continuous piecewise linear functions on a coarse simplicial mesh $\mathcal{T}^{H}$, we have $\delta_{j} \sim H_{j}$ and $\gamma_{\infty}(\alpha) \lesssim \max _{x \in \Omega} \alpha(x)$, 


\begin{tabular}{|c|cc|}
\hline$\hat{\alpha}$ & $\kappa\left(M_{A S}^{-1} A\right)$ & $\gamma_{\infty}(\alpha)$ \\
\hline $10^{0}$ & 5.2 & 2 \\
$10^{1}$ & 9.1 & 20 \\
$10^{2}$ & 58.1 & 200 \\
$10^{3}$ & 471 & 2000 \\
$10^{4}$ & 1821 & 20000 \\
\hline
\end{tabular}

\begin{tabular}{|c|cc|}
\hline$\hat{\alpha}$ & $\kappa\left(M_{A S}^{-1} A\right)$ & $\gamma_{\infty}(\alpha)$ \\
\hline $10^{0}$ & 5.2 & 2 \\
$10^{1}$ & 5.2 & 9.5 \\
$10^{2}$ & 5.2 & 14.2 \\
$10^{3}$ & 5.2 & 14.9 \\
$10^{4}$ & 5.2 & 15.0 \\
\hline
\end{tabular}

Table 1. Standard additive Schwarz with linear coarsening (left) and with multiscale coarsening (right) for $h=512^{-1}$ and $H=8 h$.

and so $\gamma_{\infty}(\alpha) \lesssim 1$ when $\alpha \sim 1$. When $\alpha(x) \rightarrow \infty$ for some $x \in \Omega$ then Theorem 1 suggests that linear coarsening may not be robust anymore. The numerical results in Table 1 (left) show that this is indeed the case and that $\gamma_{\infty}(\alpha)$ is a good indicator for the loss of robustness. The results in Table 1 are for $\Omega=[0,1]^{2}$ and $\alpha(x)=\hat{\alpha}$ on an "island" in the interior of each coarse element $K \in \mathcal{T}^{H}$ a distance $O(H)$ away from $\partial K$, with $\alpha(x)=1$ otherwise (for a precise description of $\alpha$ see [6, Example 5.1]). Also, there is exactly one subdomain $\Omega_{i_{j}}$ per coarse node $x_{j}^{H}$ with $\omega_{j} \subset \Omega_{i_{j}}$ (to ensure (C1)).

However, our framework leaves open the possibility of choosing the $\Phi_{j}$ to depend on $\alpha$ in such a way that $\gamma_{\infty}(\alpha)$ is still well-behaved. The next two examples give two possible ways of constructing such $\Phi_{j}$.

Example 2 (Aggregation-based Coarsening). Let $\mathcal{N}=\left\{x_{1}, \ldots, x_{n}\right\}$ be the set of fine freedoms, and let $\left\{W_{j}: j=1, \ldots, N_{H}\right\}$ be a non-overlaping partition of $\mathcal{N}$ (i.e. $\cup\left\{W_{j}: j=1, \ldots, N_{H}\right\}=\mathcal{N}$ and $W_{j} \cap W_{j^{\prime}}=\emptyset \quad \forall j \neq j^{\prime}$ ). For each $j$, we define a coefficient vector $\boldsymbol{\Phi}^{j} \in \mathbb{R}^{n}$ such that $\Phi_{p}^{j}=1$, if node $x_{p} \in W_{j}$, and $\Phi_{p}^{j}=0$ otherwise. Let $\Phi_{j} \in S^{h}(\Omega)$ be the linear finite element function with nodal values $\boldsymbol{\Phi}^{j}$. Note that although the aggregates $W_{j}$ are nonoverlapping, the supports $\omega_{j}$ of the functions $\Phi_{j}$ are. The overlap essentially consists of one layer of fine grid elements and so for quasi-uniform $\mathcal{T}^{h}$ we have $\delta_{j} \sim h$. In [10] (see also [2]) we go on to smooth these functions by using a simple damped Jacobi smoother. This increases the overlap. However, here we will only consider the simplest case of no smoothing.

It follows immediately from the above construction that the $\Phi_{j}$ are linearly independent and satisfy $(\mathrm{C} 2)$ and $(\mathrm{C} 3)$. Therefore, if the covering $\left\{\Omega_{i}\right\}$ is chosen such that $(\mathrm{C} 1)$ is satisfied, then Theorem 1 implies

$$
\kappa\left(M_{A S}^{-1} A\right) \lesssim \gamma_{\infty}(\alpha) \max _{j=1}^{N_{H}} \frac{H_{j}}{h} .
$$

The $\Phi_{j}$ have nonzero gradient only in the overlap of $\omega_{j}$ and so, provided $\alpha$ is well-behaved in the overlap, $\gamma_{\infty}(\alpha)$ can be bounded independent of $\max _{x \in \Omega} \alpha(x)$. In [10] we present an algorithm to choose aggregates $W_{j}$ which can be proved to satisfy this for certain choices of "binary" coefficient functions $\alpha$ by using the idea of strong connections in $A$ from algebraic multigrid 
(AMG). Given an aggregation "radius" $r \in \mathbb{N}$ and a threshold for strong connections, roughly speaking each of the aggregates $W_{j}$ is calculated by finding the strongly-connected graph $r$-neighbourhood of a suitably chosen seed node $x_{j}^{H} \in \mathcal{N}$. The aggregation procedure in [10] uses an advancing front in the graph induced by $A$ to choose good seed nodes. We refer to [10] for details and numerical results with binary and random media.

Example 3 (Multiscale Finite Element Coarsening I). Let $\mathcal{T}^{H}$ be a shaperegular mesh of coarse simplices on $\Omega$ with a typical element of $\mathcal{T}^{H}$ being the (closed) set $K$, which we assume to consist of the union of a set of fine grid elements $\tau \in \mathcal{T}^{h}$. Also, let $\left\{x_{j}^{H}: j=1, \ldots, N_{H}\right\}$ be the set of nodes of $\mathcal{T}^{H}$ and let $\mathcal{F}^{H}$ denote the set of all (closed) faces of elements in $\mathcal{T}^{H}$. (In the $2 \mathrm{D}$ case "faces" should be interpreted to mean "edges".) Finally, introduce also the skeleton $\Gamma=\cup\left\{f: f \in \mathcal{F}^{H}\right\}$, i.e. the set of all faces of the mesh, including those belonging to the outer boundary $\partial \Omega$.

Here, each of the coarse space basis functions $\Phi_{j}$ is associated with a node $x_{j}^{H}$ of $\mathcal{T}^{H}$. They are obtained by extending (via a discrete harmonic extension with respect to the original elliptic operator (1)) predetermined boundary data on the faces which contain $x_{j}^{H}$, into the interior of each element $K$. To introduce boundary data for each $j=1, \ldots, N_{H}$, we introduce functions $\psi_{j}: \Gamma \rightarrow \mathbb{R}$ which are required to be piecewise linear (with respect to the mesh $\mathcal{T}^{h}$ on $\Gamma$ ) and are required also to satisfy the assumptions:

$$
\begin{aligned}
& \psi_{j}\left(x_{j^{\prime}}^{H}\right)=\delta_{j, j^{\prime}}, \quad j, j^{\prime}=1, \ldots, N_{H}, \\
& 0 \leq \psi_{j}(x) \leq 1, \quad \text { and } \sum_{j=1}^{N_{H}} \psi_{j}(x)=1, \text { for all } x \in \Gamma, \\
& \psi_{j} \equiv 0 \quad \text { on all faces } f \in \mathcal{F}^{H} \text { such that } x_{j}^{H} \notin f .
\end{aligned}
$$

Using $\psi_{j}$ as boundary data, for each $j=1, \ldots, N_{H}$, the basis functions $\Phi_{j} \in \mathcal{S}^{h}(\Omega)$, are then defined by discrete $\alpha$-harmonic extension of $\psi_{j}$ into the interior of each $K \in \mathcal{T}^{H}$. That is, for each $K \in \mathcal{T}^{H},\left.\Phi_{j}\right|_{K} \in\left\{v_{h} \in \mathcal{S}^{h}(K)\right.$ : $\left.\left.\Phi_{j}\right|_{\partial K}=\left.\psi_{j}\right|_{\partial K}\right\}$ is such that

$$
\int_{K} \alpha \nabla\left(\left.\Phi_{j}\right|_{K}\right) \cdot \nabla v_{h}=0 \quad \text { for all } \quad v_{h} \in \mathcal{S}_{0}^{h}(K)
$$

where $\mathcal{S}^{h}(K)$ and $\mathcal{S}_{0}^{h}(K)$ are the continuous piecewise linear finite element spaces with respect to $\mathcal{T}^{h}$ restricted to $K$.

The obvious example of boundary data $\psi_{j}$ satisfying (M1)-(M3) are the standard hat functions on $\mathcal{T}^{H}$ restricted to the faces (edges) of the tetrahedron (triangle) $K$. However, these are not so appropriate if $\alpha$ varies strongly near the boundary $\partial K$. The oscillatory boundary conditions suggested in [8] are more useful in this case (see [6] for details).

This recipe specifies $\Phi_{j} \in S^{h}(\Omega)$ which can immediately be seen to be linearly independent and to satisfy the assumptions (C2) and (C3) (see [6]). Moreover, we have $\delta_{j} \sim H_{j}$. Therefore, if the covering $\left\{\Omega_{i}\right\}$ is chosen such that (C1) is satisfied, then Theorem 1 implies

$$
\kappa\left(M_{A S}^{-1} A\right) \lesssim \gamma_{\infty}(\alpha) .
$$


The numerical results in Table 1 (right), obtained for the test problem introduced in Example 1, show that additive Schwarz with multiscale coarsening is indeed robust even when the coarse mesh does not resolve discontinuities in $\alpha$ and that our theory accurately predicts this (cf. (6)). For more numerical results with multiscale coarsening see $[6,7]$.

\section{Coefficient-explicit Schwarz Theory II}

In practice, Assumption (C1) may be too restrictive, as it may require quite generous overlap of the subdomains (e.g. in the case of multiscale coarsening). The theory in $[6,7]$ does not require $(\mathrm{C} 1)$. However, it requires an underlying coarse mesh and is therefore not as easily applicable to other more general coarse spaces such as the aggregation-based ones in Example 2. For details and proofs on this section see [6].

Let $\mathcal{T}^{H}$ be a shape-regular coarse mesh as defined in Example 3, and for every $K \in \mathcal{T}^{H}$ let $H_{K}=\operatorname{diam}(K)$. We will now replace Assumption (C1) by

$$
\Phi_{j}\left(x_{j^{\prime}}^{H}\right)=\delta_{j, j^{\prime}}, j, j^{\prime}=1, \ldots, N_{H}, \text { and } \operatorname{supp}\left(\Phi_{j}\right) \subset \cup\left\{K: x_{j}^{H} \in K\right\} \text {. }
$$

This implies that the $\Phi_{j}$ are linearly independent and that $\mathcal{V}_{0}=\operatorname{span}\left\{\Phi_{j}\right.$ : $j=1, \ldots, N\} \subset \mathcal{V}^{h}$. However, even though we no longer need Assumption (C1) we do still need a mild assumption on the relative size of the subdomains and the coarse mesh. For shape-regular subdomains $\Omega_{i}$ we can write this as

$$
H_{K} \lesssim \operatorname{diam}\left(\Omega_{i}\right), \quad \text { for all } K \in\left\{K: K \cap \bar{\Omega}_{i} \neq \emptyset\right\} \text { and } i=1, \ldots, s,
$$

although we note that in [6] this requirement is generalised to allow highly anisotropic subdomains such as may arise in the application of mesh partitioning software. Note that $(\mathrm{C} 4)$ does not impose any direct structural relation between coarse mesh and subdomains.

The condition number estimate in this section separates robustness with respect to the coarse space from robustness with respect to the overlapping covering. We therefore introduce two robustness indicators. Analogous to $\gamma_{\infty}(\alpha)$ we first introduce a quantity which reflects how the coarse space handles the coefficient heterogeneity. However, here we measure the "energy" of the coarse space basis functions in the $L_{2}$-norm instead of the $L_{\infty}$-norm.

Definition 2. (Coarse space robustness indicator II).

$$
\gamma_{2}(\alpha)=\max _{j=1}^{N_{H}}\left\{H_{j}^{2-d}\left|\Phi_{j}\right|_{H^{1}(\Omega), \alpha}^{2}\right\} \quad \text { where } \quad H_{j}=\operatorname{diam}\left(\omega_{j}\right) .
$$

The second quantity which we introduce measures in a certain sense the ability of the subdomains $\Omega_{i}$ to handle the coefficient heterogeneity.

Definition 3. (Partitioning robustness indicator).

$$
\pi(\alpha)=\inf _{\left\{\chi_{i}\right\} \in \Pi\left(\left\{\Omega_{i}\right\}\right)}\left(\max _{i=1}^{s}\left\{\delta_{i}^{2}\left\|\alpha\left|\nabla \chi_{i}\right|^{2}\right\|_{L_{\infty}(\Omega)}\right\}\right)
$$


where $\delta_{i}$ is here the overlap for subdomain $\Omega_{i}$ and $\Pi\left(\left\{\Omega_{i}\right\}\right)$ denotes the set of all partitions of unity $\left\{\chi_{i}\right\} \subset W_{\infty}^{1}(\Omega)$ subordinate to the cover $\left\{\Omega_{i}\right\}$.

Roughly speaking, $\pi(\alpha)$ is well-behaved if there is a partition of unity whose members have small gradient wherever $\alpha$ is large. The weight $\delta_{i}^{2}$ is chosen to make $\pi(\alpha) \lesssim 1$ when $\alpha=1$.

Using these two robustness indicators and under the assumptions made in this section we can now state one of the main results from [6] (Theorem 3.9):

Theorem 2. Assume that (C1') and (C2)-(C4) hold true. Then

$$
\kappa\left(M_{A S}^{-1} A\right) \lesssim \pi(\alpha) \gamma_{2}(1)\left(1+\max _{i=1}^{s} \frac{H\left(\Omega_{i}\right)}{\delta_{i}}\right)+\gamma_{2}(\alpha) .
$$

where $H\left(\Omega_{i}\right)=\max _{\left\{K: K \cap \bar{\Omega}_{i} \neq \emptyset\right\}} H_{K}$ is the local coarse mesh diameter.

Note that, if in addition we assume $(\mathrm{C} 1)$, this bound does not reduce to the bound in Theorem 1. The results of Theorems 1 and 2 and the ways in which they are proved are genuinely different. Since in either case a slightly different set of robustness indicators is involved they provide two separate tools by which to establish the robustness of a particular coarse space. We will discuss this in more detail below.

Example 4 (Multiscale Finite Element Coarsening II). By definition the multiscale basis functions $\Phi_{j}$ constructed in Example 3 also satisfy (C1'). Therefore, if the covering $\left\{\Omega_{i}\right\}$ is chosen such that (C4) is satisfied, then Theorem 2 applies. As in [10] in the case of aggregation-based coarsening, it is shown in [6] (under some technical assumptions) that $\gamma_{2}(\alpha)$ can be bounded independently of $\max _{x \in \Omega} \alpha(x)$. Moreover, the numerical experiments in [6] show that these bounds are sharp and that the new preconditioner has greatly improved performance over standard preconditioners even in the random coefficient case.

Finally, to compare the bounds in Theorems 1 and 2 in the case of multiscale coarsening, let $\Omega_{j}=\omega_{j}$, for $j=1, \ldots, N_{H}$. This implies that $\delta_{j} \sim H\left(\Omega_{j}\right)$ and so

$$
\kappa\left(M_{A S}^{-1} A\right) \lesssim \pi(\alpha) \gamma_{2}(1)+\gamma_{2}(\alpha)
$$

However, in this case $(\mathrm{C} 1)$ also holds true and we can apply Theorem 2 to obtain $\kappa\left(M_{A S}^{-1} A\right) \lesssim \gamma_{\infty}(\alpha)$ (cf. (6)). It is not clear which of the two bounds in (6) and in (7) is sharper. Since the $\Phi_{j}$ form a partition of unity subordinate to the covering $\left\{\Omega_{j}\right\}$, we could bound $\pi(\alpha)$ by $\gamma_{\infty}(\alpha)$ and apply a trivial bound to $\gamma_{2}(\alpha)$ to obtain

$$
\kappa\left(M_{A S}^{-1} A\right) \lesssim \gamma_{2}(1) \gamma_{\infty}(\alpha)
$$

This would suggest that the bound in (6) is sharper than the one in (7). However, the inequalities which we used to obtain (8) from (7) are known to be not sharp in general, leaving open the possibility that (7) may be sharper for a particular choice of $\alpha$.

Linear algebra aspects of multiscale coarsening which also reveal a link to iterative substructuring are considered in [7]. Extensions of the methods and the theory to multiplicative, hybrid and deflation variants are also in $[6,7]$. 


\section{References}

[1] J. Aarnes and T.Y. Hou, Multiscale domain decomposition methods for elliptic problems with high aspect ratios, Acta Mathematica Applicatae Sinica, English Ser. 18 (2002), 63-76.

[2] M. Brezina and P. Vanek, A black-box iterative solver based on a twolevel Schwarz method, Computing, 63 (1999), 233-263.

[3] L.M. Carvalho, L. Giraud, and P. Le Tallec, Algebraic two-level preconditioners for the Schur complement method, SIAM J Sci Comp 22 (2001), $1987-2005$.

[4] T.F. Chan and T. Mathew, Domain Decomposition Methods, Acta Numerica 1994 (Cambridge University Press, 1994).

[5] L. Giraud, F. Guevara Vasquez and R.S. Tuminaro, Grid transfer operators for highly-variable coefficient problems in two-level non-overlapping domain decomposition methods, Numer Linear Algebra Appl 10 (2003), $467-484$.

[6] I.G. Graham, P.O. Lechner and R. Scheichl, Domain Decomposition for Multiscale PDEs, submitted, BICS Preprint 11/06, (2006), available electronically at http://www . bath.ac.uk/math-sci/BICS/.

[7] I.G. Graham, and R. Scheichl, Robust Domain Decomposition Algorithms for Multiscale PDEs, submitted, BICS Preprint 14/06, (2006), available electronically at http://www. bath.ac.uk/math-sci/BICS/.

[8] T.Y. Hou and X.-H. Wu, A multiscale finite element method for elliptic problems in composite materials and porous media, J Comp Phys 134 (1997), 169-189.

[9] J.E. Jones and P.S. Vassilevski, AMGe based on element agglomeration, SIAM J Sci Comp 23 (2001), 109-133.

[10] R. Scheichl and E. Vainikko, Additive Schwarz and aggregationbased coarsening for elliptic problems with highly variable coefficients, submitted, BICS preprint 9/06 (2006), available electronically at http://www . bath.ac.uk/math-sci/BICS/.

[11] R. Scheichl and E. Vainikko, Robust aggregation-based coarsening for additive Schwarz in the case of highly variable coefficients, in Proc. European Conference on Computational Fluid Dynamics, ECCOMAS CFD 2006 (P. Wesseling, E. Onate, J. Periaux, eds.), (2006).

[12] A. Toselli and O. Widlund, Domain Decomposition Methods Algorithms and Theory, Springer-Verlag, Berlin-Heidelberg-New York (2005).

[13] P. Vanek, J. Mandel and M. Brezina, Algebraic multigrid by smoothed aggregation for 2nd and 4th order elliptic problems, Computing 56 (1996), 179-196.

[14] W.L. Wan, T.F. Chan and B. Smith, An energy-minimizing interpolation for robust multigrid methods, SIAM J Sci Comp 21 (2000), 1632-1649.

[15] J. Xu and L. Zikatanov, On an energy minimizing basis for algebraic multigrid methods, Comput Visual Sci 7 (2004), 121-127. 